Research paper

\title{
Effect of drop in pipe wall stiffness on water-hammer speed and attenuation
}

FADI E. HACHEM, PhD, Laboratory of Hydraulic Constructions (LCH), Ecole Polytechnique Fédérale de Lausanne (EPFL),

Station 18, 1015 Lausanne, Switzerland.

Email: fadi.hachem@a3.epfl.ch (author for correspondence)

ANTON J. SCHLEISS, Professor and Director, Laboratory of Hydraulic Constructions (LCH), Ecole Polytechnique Fédérale de Lausanne (EPFL), Station 18, 1015 Lausanne, Switzerland.

Email: anton.schleiss@epfl.ch

\begin{abstract}
The effect of local drop of wall stiffness in pressurized waterways on the pressure wave speed and the wave attenuation during transients is investigated experimentally. A new signal-processing procedure to identify the presence of a weak reach is introduced and validated by physical experimentation based on assessing the pressure and vibration records acquired at both ends of a multi-reach steel test pipe. Water-hammer was generated by closing a downstream valve, whereas the weak reaches were simulated by replacing the steel portions with aluminium and PVC material. The wave speed and wave attenuation factor during transients are considered as global indicators of local and large changes in stiffness of the pipe wall. The method is capable of locating the stiffness weakness along the test pipe if one PVC reach is used. The error in estimating the position of such a reach relative to the real position of its mean value varies up to $23 \%$.
\end{abstract}

Keywords: Geophone, monitoring, steel-lined tunnel, wall stiffness, water-hammer, wave attenuation, wave speed

\section{Introduction}

Unsteady flows in pressurized water systems offer a challenge in computation, visualization and analysis, particularly in the presence of system facilities and features such as elbows, valves or junctions. The interaction between the structural wall and the confined water influences the transient flow behaviour providing signatures of specific characteristics along the conduit (Bergant et al. 2008a). The changes in transient pressures generated by a wall-leaking crack have been extensively studied using hydraulic-based monitoring techniques (Ferrante and Brunone 2003, Covas et al. 2005, Hunaidi 2006). A different structural wall aspect was studied by Stephens et al. (2008) to estimate the location of internal wall damage of a composite concrete-steel pipeline based on a transient model combined with a generic algorithm and field measurements. It is also known that a local difference of the wall characteristic impedance as a function of wave speed and flow area relative to the rest of the waterway system generates hydro-acoustic boundaries that create wave reflections and transmissions thereby changing the wave speed.

The alteration of the water-hammer wave speed and wave attenuation induced by substituting certain reaches of the steel test pipe with others of material as aluminium or PVC, called "weak reaches" below, have been investigated experimentally. Transient signals of pressure and vibration are acquired at the both ends of the pipe and then scrutinized using new processing methods. The proposed procedure applies practically for monitoring of steel-lined pressure shafts and tunnels to identify the local deterioration of the backfill concrete or the rock zone surrounding the liner. The deteriorated multilayer system (steelconcrete-rock) that composes the wall of these structures is experimentally simulated by a weaker aluminium or PVC reach.

\section{Theoretical background}

\subsection{Mass and momentum equations}

The water-hammer phenomenon in pressurized schemes is modelled using the classical mass and momentum equations for one-dimensional flows as (Wylie et al. 1993)

$$
\begin{array}{r}
\frac{\partial q}{\partial x}+\frac{g \pi D^{2}}{4 a^{2}} \frac{\partial h}{\partial t}=0 \\
g \frac{\partial h}{\partial x}+\frac{4}{\pi D^{2}} \frac{\partial q}{\partial t}+\frac{4}{\rho_{w} D} \tau_{w}=0
\end{array}
$$

Revision received 9 January 2012/Open for discussion until 31 October 2012. 
in which $q(x, t)$ is the discharge, $h(x, t)$ the piezometric head, $g$ the gravitational acceleration, $D$ the internal pipe or tunnel diameter, $a$ the water-hammer wave speed, $\rho_{w}$ the water density, $x$ the spatial coordinate along the longitudinal axis, $t$ the time and $\tau_{w}$ the shear stress at the water-wall interface.

\subsection{Water-hammer wave speed}

The quasi-static wave speed without fluid-structure interaction (FSI) follows from, e.g. Wylie et al. (1993). The wave speed then becomes frequency-dependent and other mathematical models than presented above should be used (Lavooij and Tijsseling 1991, or Tijsseling 2007). Between the quasi-static and the FSI cases, transient models similar to those proposed by Covas et al. (2004) consider the pipe wall as a linear-viscoelastic material.

In steel-lined pressure tunnels under plain strain conditions, linear elasticity and small deformations considering neither FSI nor the dynamic or creep effects of the tunnel wall, the waterhammer wave speed is

$$
a=\sqrt{\frac{1}{\rho_{w}\left(1 / K_{w}+(2 / \eta) \cdot\left(\mathrm{d} u_{r}^{s}(r) / \mathrm{d} p\right)\right)}}
$$

where $\mathrm{d} u_{r}^{s}(r) / \mathrm{d} p$ is the first derivative of the radial displacement of the steel liner $u_{r}^{s}$ relative to the internal pressure $p$ at the waterliner interface of radius $r_{i}$ and $K_{w}$ is the bulk modulus of water. The $\mathrm{d} u_{r}^{s}(r) / \mathrm{d} p$ ratio is a constant that depends on the geometrical and mechanical characteristics of the steel liner and the state (cracked or un-cracked) of the surrounding backfill concrete and rock mass (Hachem and Schleiss 2011). The bulk modulus and density of water and, thus, the wave speed are influenced by the presence of air and sediments in the water. By ignoring the presence of air in the flow and considering as basic configuration the actual state of pressure tunnel at the beginning of monitoring, the velocity of a pressure wave travelling between two crosssections of the tunnel will be affected by the local increase in its cross-sectional area or/and the decay of the radial wall stiffness. The increase in the cross-sectional area is induced by the yielding of the steel liner of low-to-moderate strength, while the stiffness decay is a consequence of progressive deterioration of the backfill concrete and the near-field rock mass. The drop of wall stiffness can be generated in both low- and high-strength steel liners and is considered as the first indicator of a possible future liner break. Therefore, a reliable and continuous estimation of the wave speed is crucial to detect the formation of weak reaches of low wall stiffness along pressure shafts or tunnels.

\subsection{Wave attenuation}

The attenuation of water-hammer wave along a pressurized scheme is caused by energy dissipation induced by many factors such as surge control devices, the presence of dissolved air in water, the shear stress effect and the inelastic behaviour of the pipe or shaft wall, leakage and the FSI (Bergant et al. 2008a, 2008b). The inelastic behaviour is important in plastic pipes and related to the mechanical properties of the pipe wall and to the fluid wave frequency (Hachem and Schleiss 2011). The wall shear stress $\tau_{w}$ is the summation of a quasi-steady and an unsteady friction component. The former is estimated using the Darcy-Weisbach or the Hazen-Williams formulae, whereas the latter results from empirically-based models such as in Bergant et al. (2001) or from physically-based models such as in Vardy et al. (1993). The wave dissipation due to steady or unsteady friction becomes important for high friction factors or long pipes of small internal diameter (Ghidaoui et al. 2005).

The attenuation of a water-hammer wave can also be induced by wave transmission and reflection phenomena encountered at the boundaries of the weak reaches. This boundary effect alters particularly the front of the wave crossing these reaches. For a frictionless liner wall, at the $n$th boundaries of the weak reaches, the incident wave will have a magnitude of (Wylie et al. 1993)

$$
\frac{h_{n}-h_{0}}{h_{w}-h_{0}}=2^{n} \prod_{j=1}^{n} \frac{1}{1+a_{j+1} A_{j} / a_{j} A_{j+1}}
$$

where $h_{n}$ is the magnitude of the transmitted wave crossing the $n$th boundary, $h_{w}$ is the magnitude of the incident wave before reaching the weak reach, $h_{0}$ is the steady-state piezometric head, $A_{j+1}$ and $A_{j}$ are the cross-sectional areas and $a_{j+1}$ and $a_{j}$ are the wave speeds in reaches $(j+1)$ and $(j)$, respectively. Therefore, the formation of a weak reach somewhere along pressure shafts or tunnels induces additional attenuation of pressure waves due to the higher damping coefficient of the weak reach wall and the wave reflection/transmission at its boundaries. Herein, the latter attenuation type is discussed and will be considered as the second indicator of the existence of a weak reach.

\section{Experimentation}

\subsection{Set-up description}

The experimental facility shown in Fig. 1 is a closed hydraulic loop designed to generate water-hammer transients inside a multi-reach steel test pipe of $150 \mathrm{~mm}$ internal diameter and length

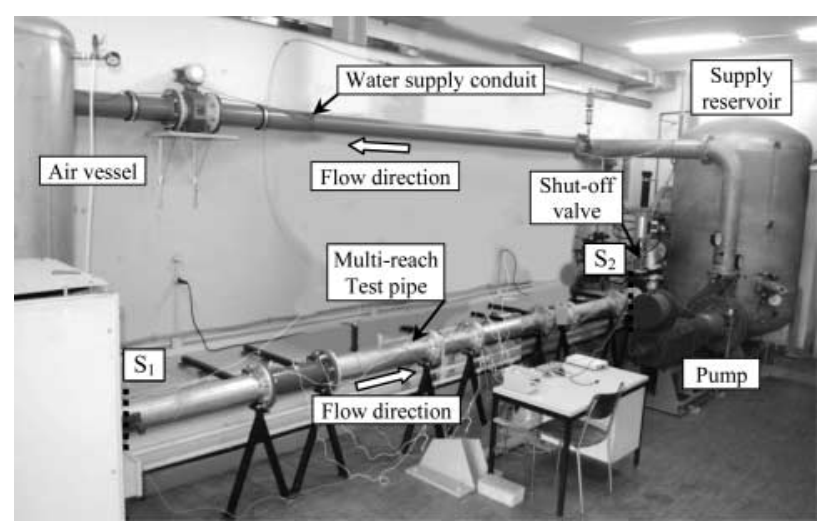

Figure 1 View of experimental facility 


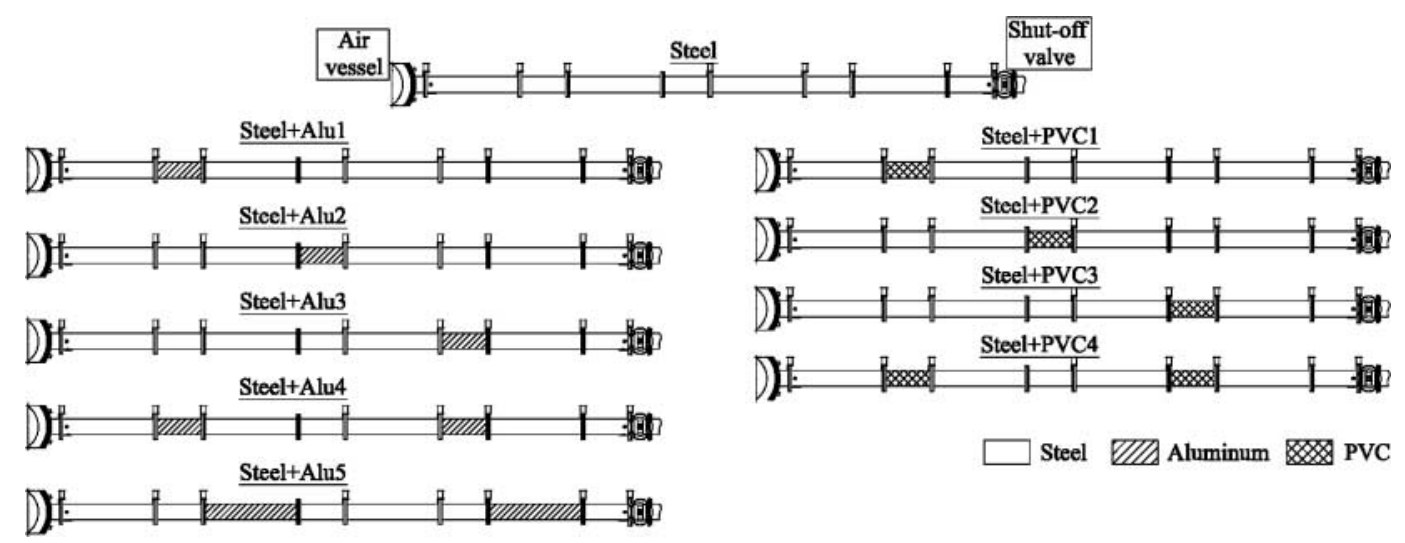

Figure 2 Ten different configurations of test pipe

of $6.25 \mathrm{~m}$ measured from the shut-off valve to the upstream air vessel. The shut-off valve is followed by a purge valve, two elbows, an elastic joint and a control valve located at the entrance of the supply reservoir. The total length of the test pipe comprising these elements is about $2 \mathrm{~m}$. The test pipe is divided into several reaches of 0.5 and $1.0 \mathrm{~m}$ length fitted together with flanges of $285 \mathrm{~mm}$ external diameter and a thickness of $24 \mathrm{~mm}$. The flanges are also used to rigidly fix the test pipe along its length to minimize longitudinal and transverse movements. The weak reaches were modelled using pipe wall material of different $(E \cdot e)$ values than the rest of the test pipe, where $E$ is the Young modulus and $e$ the thickness of the pipe wall. Aluminium and PVC reaches of $E \cdot e=345$ and $15 \mathrm{MN} / \mathrm{m}$, respectively, were used. This consists of a local drop of pipe wall stiffness relative to steel $(E \cdot e=945 \mathrm{MN} / \mathrm{m})$ of about $63 \%$ for aluminium and nearly $98 \%$ for PVC. A total of 120 tests have been carried out on the 10 test pipe configurations shown in Fig. 2. For each configuration, 12 repetitive tests were performed. An initial steady-state flow around $65 \mathrm{l} / \mathrm{s}$ with a flow velocity of $3.7 \mathrm{~m} / \mathrm{s}$, a Reynolds number of 462,500 and a mean pressure of 0.21 bar were maintained for all tests. The water-hammer was generated by closing the downstream guillotine valve which is activated by an air jack equipped with input and output air electro-valves. Their mean closure times were $0.235 \mathrm{~s}$ which is considered slow relative to the characteristic time of the test pipe equal to $0.013 \mathrm{~s}$.

(a)

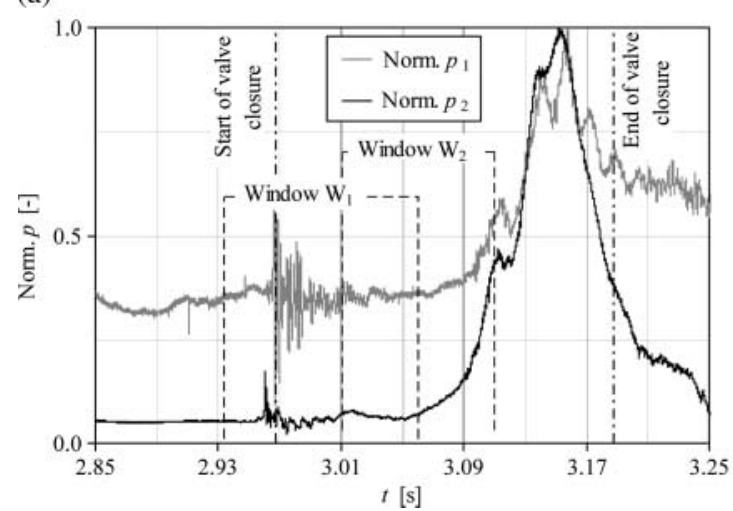

The volume of the air needed to activate the jack was provided by an air compressor of 10 bar constant pressure. The opened and closed states of the shut-off valve were detected by two infrared diffusive sensors. On the highest conduit point, an air purge valve was installed to evacuate the captured air inside the test rig. The data acquisition system includes: (i) eight pressure transducers (HKM-375M-7-BAR-A, Kulite ${ }^{\circledR}$ ) with a pressure range of \pm 7 bar and an accuracy of $0.5 \%$, (ii) two geophones (SM-6/SB, $4.5 \mathrm{~Hz}, 3500 \Omega$, SENSOR ${ }^{\circledR}$ Nederland b.v.) with a sensitivity of $78.9 \mathrm{~V} /(\mathrm{m} / \mathrm{s})$ and a tolerance of $\pm 5 \%$, (iii) a NI-USB-6259 acquisition card $\mathrm{M}$ series with 32 analogue input channels and 2 analogue output channels to activate the two electro-valves of the shut-off valve and (iv) a personal computer. The water-hammer pressures $p_{1}$ and $p_{2}$, and the output geophone signals $V_{1}$ and $V_{2}$, were recorded at sections $S_{1}$ and $S_{2}$ located at the ends of the test pipe (Fig. 1) at a sampling frequency of $f_{s}=20 \mathrm{kHz}$.

\subsection{Data analysis}

\subsubsection{Estimation of wave speed based on pressure data}

The wave speed was estimated by processing the water-hammer pressure and the radial vibration of the pipe wall at sections $S_{1}$ and $S_{2}$. Typical time histories of normalized pressures and geophones outputs are shown in Fig. 3. The pressure signals inside window $\mathrm{W}_{1}$ were generated at the start of the valve closure by the (b)

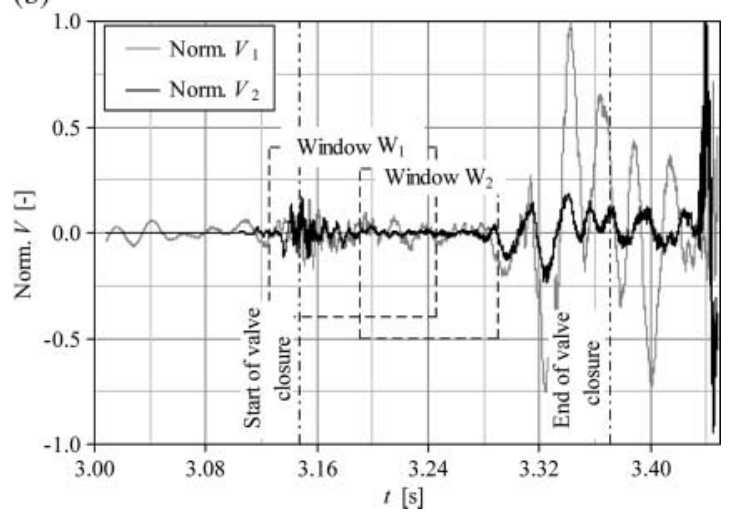

Figure 3 Typical records of measured transient records at up- and downstream sections $\mathrm{S}_{1}$ and $\mathrm{S}_{2}$ (a) pressures, (b) geophone outputs $\mathrm{W}_{1}$ and $\mathrm{W}_{2}$ 
external pressure excitation caused by the air impact inside the valve jack. These pressure fluctuations die out after $0.045 \mathrm{~s}$ from valve closure start, defining the lower time border of a second window $\mathrm{W}_{2}$, in which the progressive flow stop by the valve generated water-hammer pulsations inside the test pipe. The upper time border $\mathrm{W}_{2}$ was fixed after $0.16 \mathrm{~s}$ from valve closure start. Beyond this time, a series of no flow tests revealed the presence of an important pressure drop followed by high-amplitude and high-frequency fluctuations. The former was probably caused by the compression and expansion of an air pocket inside the upper valve cover, whereas the latter was generated by the deformation, friction and vibration of the internal valve core. The pressure signals of $0.12 \mathrm{~s}$ duration acquired within window $\mathrm{W}_{1}$ have been analysed by Hachem and Schleiss $(2010,2012)$ where a new method for detecting the presence, location and severity of a local weak reach was proposed. The pressure signals inside window $\mathrm{W}_{2}$ do not have a clear wave front that is easily identified and followed as in their first parts. Therefore, a new procedure to estimate the wave speed from the pressure records inside $\mathrm{W}_{2}$ is proposed herein and validated by a series of experimental tests.

This procedure decomposes each signal $p_{1}$ and $p_{2}$ into an approximation $A_{J}$ and several detail $D_{i}$ components using the wavelet decomposition technique (Mallat 1990, Ferrante and Brunone 2003, Hachem and Schleiss 2012) using the Daubechies (db10) mother wavelet. The details $D_{4}$ to $D_{7}$ for each pressure signal are then summed together to construct two new signals $S\left(p_{1}\right)$ and $S\left(p_{2}\right)$, filtering the signals with a band-pass filter of low and high frequencies of 72 and $486 \mathrm{~Hz}$, respectively. These were selected to preserve the information of the incident-reflection waves between the supply reservoir and the air vessel $\left(=a_{\text {steel }} /\left(2 L_{\max }\right)=1245.4 /(2 \cdot 8.25)=75.5 \mathrm{~Hz}\right)$, and between the closest weak reach boundary to the air vessel and this latter $\left(a_{\text {steel }} /\left(2 L_{\min }\right)=1245.4 /(2 \cdot 1.29)=482 \mathrm{~Hz}\right)$. The signals $S\left(p_{1}\right)$ and $S\left(p_{2}\right)$ of the pressure records of Fig. 3(a) are shown in Fig. 4(a).

The procedure is continued by computing the energy content history of signal $S\left(p_{2}\right)$ using the floating root-mean-square
(RMS) given by

$$
\mathrm{RMS}_{j}=\sqrt{\frac{1}{N} \sum_{i=j}^{j+N-1} p_{i}^{2}}
$$

in which $p_{i}$ is the discrete pressure values of signal $S\left(p_{2}\right)$, and $N$ is a real parameter defining the resolution of the RMS and represents the number of pressure points inside a one period interval. High-frequency $f_{\max }=500 \mathrm{~Hz}$ is used to determine the value of $N$ according to $N=\left(1 / f_{\max }\right) \cdot f_{s}=(1 / 500) \cdot 20,000=40$. The RMS curve shows positive shifts at times when an important pressure energy package crosses section $\mathrm{S}_{2}$. A RMS-threshold line of 0.01 bar was chosen to define a portion or a signature of the $S\left(p_{2}\right)$ signal. This signature is limited by the times of the two minimum peaks of the first RMS lobe with a positive shift that crosses the threshold line. The RMS-curve, the energy threshold line, and the signal borders of $S\left(p_{2}\right)$ given in Fig. 3(a) are shown in Fig. 4(b).

The procedure ends with a cross-correlation between the signature of pressures $S\left(p_{2}\right)$ and $S\left(p_{1}\right)$. For discrete functions, the cross-correlation is defined as (Lang 1987)

$$
\left(s\left(p_{1}\right) * S\left(p_{2}\right)\right)[n]=\sum_{m=1}^{s} S\left(p_{1}\right) *[m] \cdot S\left(p_{2}\right)[n+m]
$$

where $S\left(p_{1}\right)^{*}$ is the complex conjugate of $S\left(p_{1}\right), l$ and $s$ are the lower and upper limits of the signal time interval, respectively, and $n$ and $m$ are the two positive integers. The estimated traveltime of the water-hammer wave speed between the two sections $\mathrm{S}_{1}$ and $\mathrm{S}_{2}$ corresponds to the time-lag at the maximum positive peak of the cross-correlation curve identified between two time borders obtained from the logical wave speed values of 1400 and $800 \mathrm{~m} / \mathrm{s}$ according to the relations $t_{\text {Lower border }}=5.88 / 1400=$ $0.0042 \mathrm{~s}, t_{\text {Higher border }}=5.88 / 800=0.00735 \mathrm{~s}$. The peak time of the signals shown in Fig. $4(\mathrm{a})$ is $t_{p 2, p 1}=0.0059 \mathrm{~s}$ and easily transformed to the wave speed according to

$$
a=\frac{L_{p 2, p 1}}{t_{p 2, p 1}}
$$

(b)
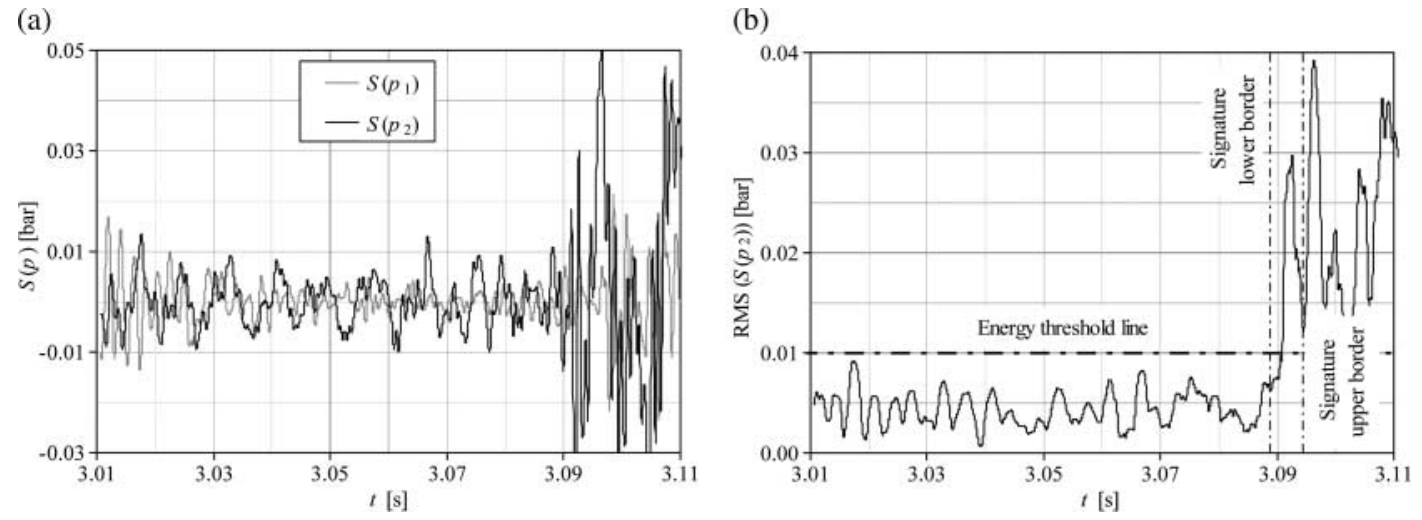

Figure 4 (a) Pressure signals constructed by summing wavelet details $D_{4}$ to $D_{7}$, (b) floating RMS of $S\left(p_{2}\right)$ signal shown in (a) including energy threshold line and borders of $S\left(p_{2}\right)$ pressure signature 
(a)

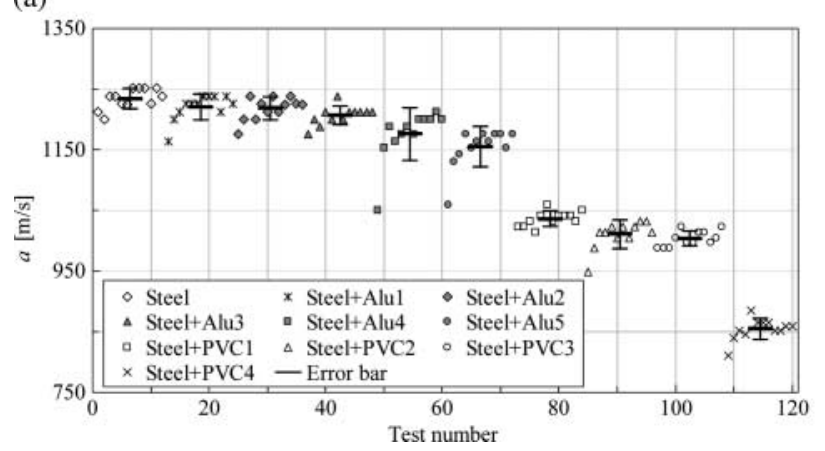

(b)

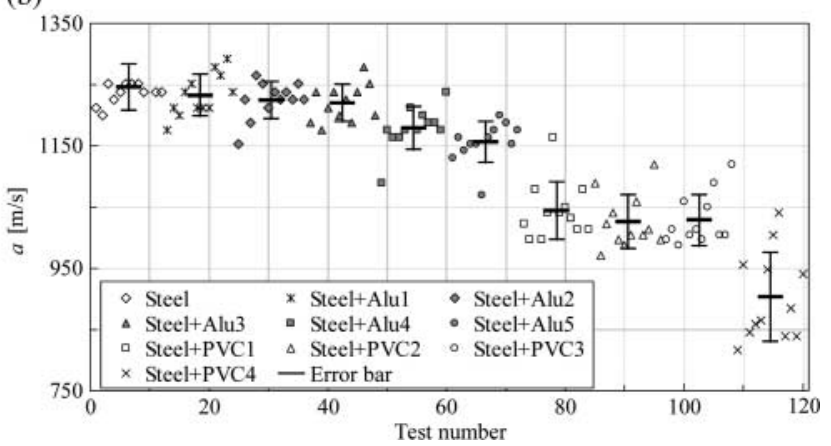

Figure 5 Water-hammer wave speed estimated from pressure records for (a) steep wave front as at window $\mathrm{W}_{1}$ of Fig. 3(a), (b) waves generated by progressive flow stop by shut-off valve as at window $\mathrm{W}_{2}$ of Fig. 3(b)

(a)

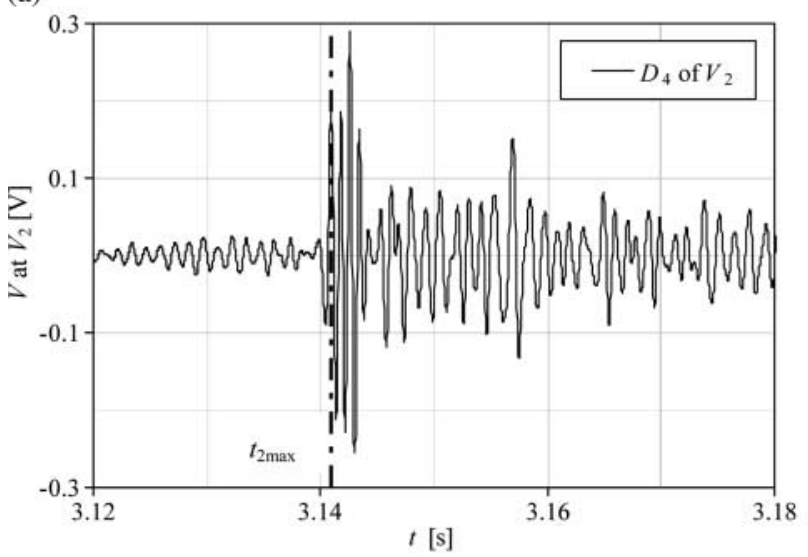

(b)

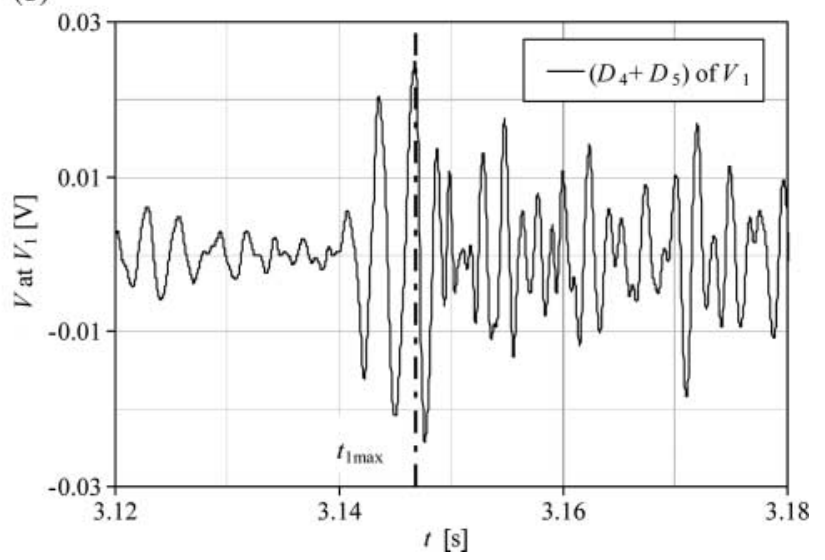

Figure 6 Wavelet details components of geophone output signals shown in window $\mathrm{W}_{1}$ of Fig. 3(b) (a) $D_{4}$ at section $\mathrm{S}_{2}$, (b) $\left(D_{4}+D_{5}\right)$ at section $\mathrm{S}_{1}$

where $L_{p 2, p 1}$ is the distance of $5.88 \mathrm{~m}$ separating the two pressure sensors $p_{1}$ and $p_{2}$.

The estimated wave speeds between sections $S_{1}$ and $S_{2}$ determined according to Hachem and Schleiss (2012) for pressure signals at window $\mathrm{W}_{1}$ and obtained according to the above procedure for signals at window $\mathrm{W}_{2}$ are compared in Fig. 5. These wave speeds are given for all 120 tests conducted on the 10 test configurations. The means and standard deviations of each 12 tests that correspond to each pipe configuration are also shown. A good agreement is found between mean values estimated by the two pressure-based methods with a maximum relative difference of $5.8 \%$ for configuration "steel + PVC4".

\subsubsection{Estimation of wave speed based on geophone data}

The radial vibrations of the pipe wall were determined using the two geophones fixed on the exterior pipe surface at sections $S_{1}$ and $S_{2}$. The geophone is a simple sensor that has a suspended moving coil around a permanent magnet. As the coil moves relative to the magnet, a voltage is induced in the coil according to Faraday's law. The induced voltage is proportional to the relative coil-magnet velocity with the proportionality factor as sensitivity $G$. This sensor type is cheap, passive (no need for power supply) and has good linearity. It is usually used in seismologic surveys to detect refraction and/or reflection from subsurface formations after generating a surface disturbance by explosives. It has been also used in leak detection monitoring of shallow underground pipelines by allowing users to scan the entire length of the inspected pipelines and to record the noise being reflected by the leak (Mays 2000). Hunaidi (2006) developed an acoustic method based on cross-correlating two accelerometer signals to determine the wave speed in a water distribution pipe reach to extract its mean wall thickness from standard wave speed formulae. The advantage of the method developed herein consists of using geophones instead of relatively expensive accelerometers and signal conditioning hardware. The wave speed values obtained from processing the geophone output signals $V_{1}$ and $V_{2}$ measured at window $\mathrm{W}_{1}$ (Fig. $3 \mathrm{~b}$ ) are compared with those estimated from pressure signals.

The analysis starts by decomposing the two geophone signals using the Daubechies (db10) mother wavelet. Figure 6(a) shows the time history of detail $D_{4}$ for the geophone output $V_{2}$ of Fig. 3(b), indicating a significant amplitude increase at time $t_{2 \max }$ as the wave front crosses pipe section $\mathrm{S}_{2}$. This signal detail is transformed to pipe surface displacement $u$ according to the transfer function $H(f)$ obtained from the second-order differential equation of motion of the damped mass of the geophone sensor

$$
H(f)=\frac{\operatorname{FFT}(v[\mathrm{~V}])}{\operatorname{FFT}(u[m])}=\frac{-2 \pi i f^{3} G}{f_{0}^{2}-f^{2}+2 i \xi f_{0} f}
$$


(a)

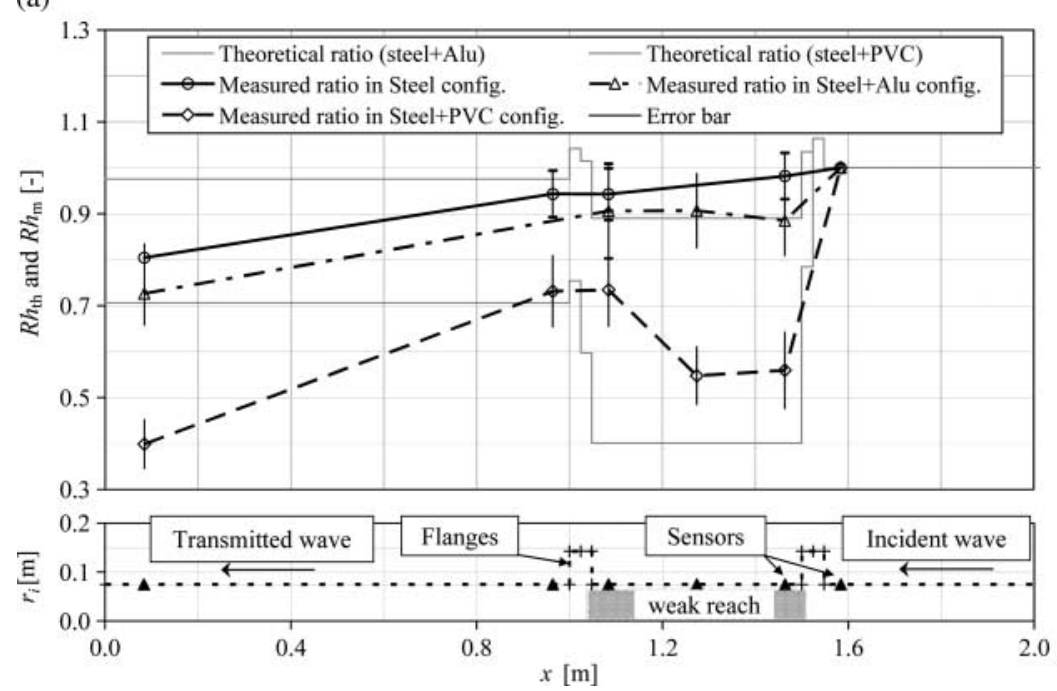

(b)

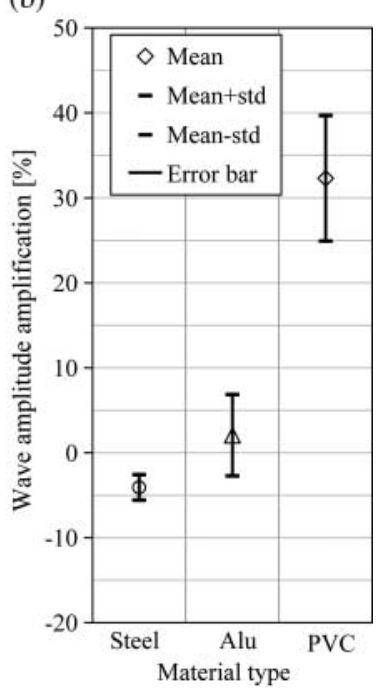

Figure 7 (a) Local theoretical and experimental attenuation ratios of pressure wave front of weak reach, (b) means and standard deviations of amplifications of wave front magnitude inside weak reach for different materials

where FFT is the Fast Fourier Transform, $v$ the geophone output signal in $(\mathrm{V}), \mathrm{i}=(-1)^{1 / 2}$ is the complex number, $f$ the frequency, and $f_{0}(=4.5 \mathrm{~Hz})$ and $\xi(=0.58)$ are the natural frequency and the damping ratio of the geophone, respectively. The maximal computed radial displacement at time $t_{2 \max }$ is compared with the value of the pipe wall determined from the pipe formula involving the wall characteristics at section $\mathrm{S}_{2}$ and the pressure increase at the wave front. This comparison indicates a relative mean error of $12 \%$ and proves that detail $D_{4}$ contains the most important portion of the radial component of the pipe wall displacement. At section $\mathrm{S}_{1}$, the sum $\left(D_{4}+D_{5}\right)$ identifies the arrival time $t_{1 \max }$ of the water-hammer pressure wave (Fig. 6b). Time $t_{1 \max }$ is determined from the first peak time of record $\left(D_{4}+D_{5}\right)$ resulting in $a$ between 800 and $1400 \mathrm{~m} / \mathrm{s}$ computed from Eq. (7) with $t_{p 2, p 1}=\left(t_{1 \max }-t_{2 \max }\right)$. The comparison between the wave speeds estimated by the proposed geophone-based method and the pressure approach for the five test configurations agrees well with a maximum relative difference of $9.3 \%$. The wave speeds obtained from the geophone signals for the "steel" configuration are around $80 \mathrm{~m} / \mathrm{s}$ higher than those estimated from pressure measurements. The deviations in water-hammer wave speed for the "steel" configuration is probably caused by the interference of geophones with the stress waves travelling at high speed inside the test pipe wall which are considerably attenuated by the presence of the PVC reach along the pipe. Note that the geophone signals inside window $\mathrm{W}_{2}$ were not analysed by the herein proposed method due to the long dissipation time of the output signal generated in window $\mathrm{W}_{1}$.

\subsubsection{Estimation of wave attenuation induced by the weak reach boundaries}

For the front wave generated in window $\mathrm{W}_{1}$, a total of six pressure sensors were used to measure the water transients inside and at the up- and downstream sides of the aluminium and PVC reaches. Three sensors were placed inside the weak reach (one in the middle and two $60 \mathrm{~mm}$ from each of its end), one upand two downstream from it (Fig. 7(a)). For each "steel + PVC" and "steel + Alu" configurations, the six sensors were displaced to follow the position of the weak reach along the pipe. The pressure sensors at sections $S_{1}$ and $S_{2}$ were used to record the global wave attenuation along the pipe. Figure 7(a) shows the theoretical $R h_{\text {th }}$ and measured pressure head ratios $R h_{\mathrm{m}}$ between the transmitted and incident waves, as defined in Eq. (4) for three different reaches made of steel, aluminium and PVC material.

In the presence of the PVC reach, the measured results show that the front wave looses around $44 \%$ of its magnitude when entering the weak reach. The reflection from the downstream border amplifies the wave front by some 32\% (Fig. 7(b)). The wave continues its downstream propagation with an attenuation slope higher than for the steel and aluminium cases. The measured ratios inside the weak reach are $20 \%$ higher than the theoretical values from Eq. (4). The higher downstream slope and the differences between the theoretical and measured pressure ratios are probably caused by the inelastic behaviour of the PVC reach. For the aluminium reach, the same wave behaviour is observed with less attenuation and less amplification when entering and leaving the reach, respectively. The additional wave attenuation induced by the aluminium and PVC reaches is confirmed by the total attenuation factor of the entire test pipe.

Figure 8 shows the relative attenuation ratios of the front wave pressure $\Delta h$ between $\mathrm{S}_{1}$ and $\mathrm{S}_{2}$ for all test configurations. Significant and proportional differences of $+20 \%$ in "steel + PVC1, 2, 3" and +35\% in "steel + PVC4" between the "steel" and the "steel + PVCs" configurations occur, which are absent in the "steel + Alu" cases. Note that it was difficult to extract similar information regarding the wave attenuation by using the geophones or the pressure records at window $\mathrm{W}_{2}$. 


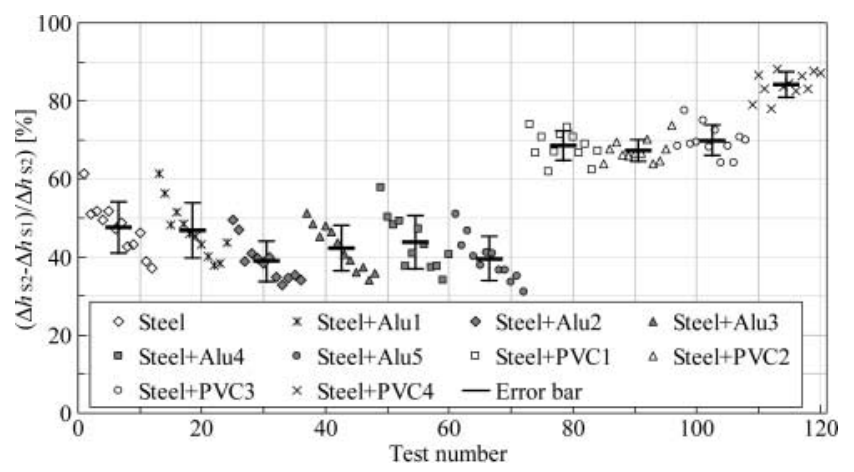

Figure 8 Relative attenuation ratios between sections $S_{1}$ and $S_{2}$ estimated from pressure records having a steep wave front

\subsubsection{Weak reach location}

The incident-reflection travel times between the weak reach and the air vessel are estimated using FFT of the pressure records at Window $\mathrm{W}_{2}$. The records of the test configurations with aluminium reach are similar to those for the "steel" configuration due to the small wave magnitude reflected from the aluminium reach boundaries. Therefore, the localization of these reaches using the FFT approach is not possible. For each "steel + PVC" test pipe configuration, the mean of the normalized FFT (with Hanning windowing) at $S_{1}$ and $S_{2}$ was computed. The normalization was obtained by dividing the FFT magnitudes by that at $80 \mathrm{~Hz}$, corresponding to the incident-reflection travel time between the supply reservoir and the air vessel. Figure 9 shows the average curves of the normalized FFT for the $p_{1}$ and $p_{2}$ signals acquired from the "steel" and "steel + PVCs" configurations. The curves "steel + PVCs" show significant differences in their pattern compared with the "steel" configuration resulting from the pressure reflection generated by the weak reach inside the test pipe. The frequency that corresponds to the weak reach for each pipe configuration is identified after discarding the FFT peaks of the "steel + PVCs" records of identical frequencies as the peaks of the "steel" configuration (grey bands in Fig. 9). For "steel + PVC1", one of the many $p_{1}$ FFT peaks (marked by circles in Fig. 9) should be chosen. This was done by using couples of $p_{1}$ and $p_{2}$ peak frequencies to compute the fundamental frequency $f_{\text {fund }}$ according to

$$
f_{\text {fund }}=\frac{f_{\max , p_{1}} \cdot f_{\max , p_{2}}}{f_{\max , p_{1}}+f_{\max , p_{2}}}
$$

where $f_{\max , p 1}$ and $f_{\max , p 2}$ are the FFT maximum peaks of $p_{1}$ and $p_{2}$, respectively. The frequencies $f_{\text {fund }}$ were then compared with the theoretical value $a_{\text {steel }} /\left(2 L_{\max }\right)=75.5 \mathrm{~Hz}$. The pair of frequencies which gives the nearest $f_{\text {fund }}$ relative to the theoretical value was retained. For the "steel + PVC1" configuration, these frequencies are $f_{\max , p 1}=320 \mathrm{~Hz}$ and $f_{\max , p 2}=120 \mathrm{~Hz}$, whereas for "steel + PVC2" and "steel + PVC3", the only remaining frequencies of the FFT $p_{1}$ records after discarding peak frequencies of the "steel" configuration are 210 and $140 \mathrm{~Hz}$, respectively. For these, Eq. (9) has not been used to identify the frequency peaks.
The estimation of the incident-reflection travel distance $L_{1}$ between the weak reach and the air vessel was done by using the mean wave speed of the "steel" configuration $\left(a_{\text {steel }}=\right.$ $1245.4 \mathrm{~m} / \mathrm{s}$ ) and the identified fundamental frequencies from the FFT of $p_{1}$. The travel distances and the errors relative to the real path lengths are given in Table 1. The error in estimating the position of the weak reach relative to the real position of its middle section varies from $2.3 \%$ to $22.7 \%$. A step-by-step flow chart diagram of the analysis procedure used for the physical tests is given by Hachem (2011).

\section{Conclusions}

A new procedure for estimating the wave speed and the wave attenuation inside pressurized schemes during water-hammer phenomena in the presence of a local drop of wall stiffness is proposed. This procedure was validated by a series of 120 experimental tests conducted in a multi-reach steel pipe where the weak reaches were physically modelled by exchanging the steel reaches by aluminium and PVC. It is based on acquiring and processing pressure and vibration records obtained at two sections of the test pipe. This method also estimates the position of the weak reach if only one PVC reach is used. It includes the wavelet decomposition and filtering techniques and the FFTs. The following items were found:

(1) Wave speeds computed by using the proposed procedure from both the pressure and geophone sensors agree well with these from pressure records in previous tests using steep wave fronts. A maximum relative mean error of $6 \%$ was observed in the "steel + PVC4" configuration if the pressure records are used to estimate the wave speed. The processing of the geophone signals gives a similar mean error but overestimates the wave speed values in the "steel" configuration by about $80 \mathrm{~m} / \mathrm{s}$.

(2) The method is able to determine the wave attenuation factor by using only the steep front wave pressures. Significant and proportional attenuation differences of $+20 \%$ in the "steel + PVC1, 2, 3" and $+35 \%$ in the "steel + PVC4" relative to the "steel" configuration were observed.

(3) The wave speed and wave attenuation factor during transients can be considered global indicators of local and large changes in stiffness of the pipe wall.

(4) Using FFT analysis for the pressure signals one weak reach of very low stiffness relative to the steel was localized. The error in estimating the position of such a reach relative to the real position of its middle varies up to $23 \%$.

The monitoring procedure consists on acquiring continuously the transient pressure signals. Once a significant and persistent decrease in the wave speed and an increase in the wave attenuation are detected, a drop of the wall stiffness is suspected to occur somewhere along the shaft. The state of the shaft with a weak reach will be then considered as the basic configuration for 

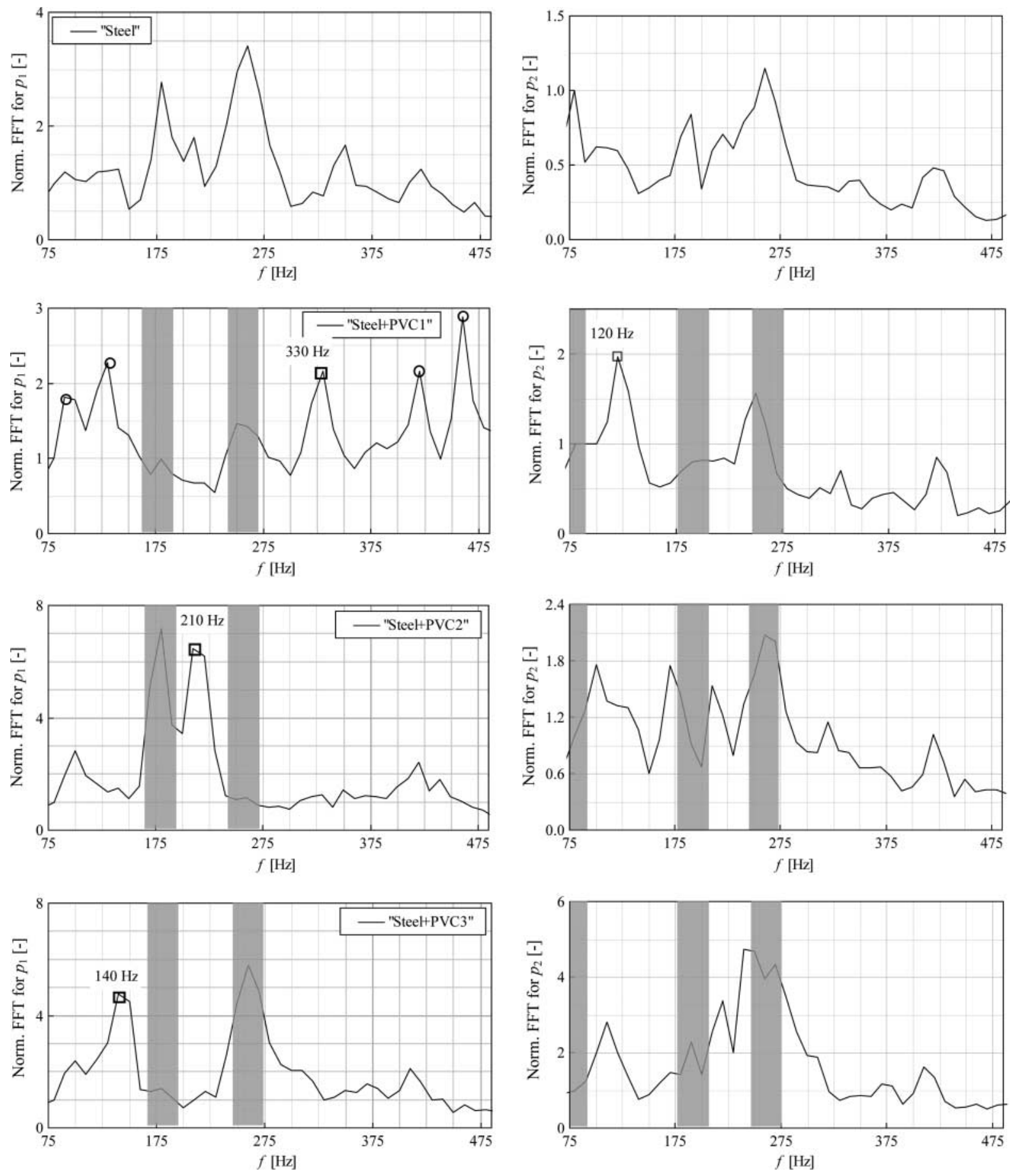

Figure 9 Normalized FFTs of pressures $p_{1}$ and $p_{2}$ inside window $\mathrm{W}_{2}$ of Fig. 3(a) for "steel" and "steel + PVCs" test configurations

Table 1 Estimated distances between middle of weak reach and sensor $p_{1}$ obtained from FFT approach for three test pipe configurations "steel + PVC1, 2,3"

\begin{tabular}{|c|c|c|c|c|c|c|}
\hline $\begin{array}{l}\text { Test pipe } \\
\text { configuration }\end{array}$ & Path & $\begin{array}{l}\text { Real path length } \\
\text { to centre of } \\
\text { WR, } L_{1}(\mathrm{~m})\end{array}$ & $\begin{array}{l}\text { Estimated } a \text { for } \\
\text { "Steel" configuration } \\
(\mathrm{m} / \mathrm{s})\end{array}$ & $\begin{array}{c}p_{1} \text { peak } \\
\text { frequency, } \\
f_{\text {max }, p 1}(\mathrm{~Hz})\end{array}$ & $\begin{array}{c}\text { Estimated } L_{1} \\
\text { path length } \\
(\mathrm{m})\end{array}$ & $\begin{array}{l}\text { Relative } \\
\text { error }(\%)\end{array}$ \\
\hline Steel + PVC1 & $p_{1}-\mathrm{WR}^{\mathrm{a}}$ & 1.54 & & 330 & 1.89 & 22.7 \\
\hline Steel + PVC2 & $p_{1}-\mathrm{WR}$ & 3.04 & 1245.4 & 210 & 2.97 & 2.3 \\
\hline Steel + PVC3 & $p_{1}$-WR & 4.56 & & 140 & 4.45 & 2.4 \\
\hline
\end{tabular}

${ }^{a}$ WR stands for Weak Reach. 
the future monitoring records. The reflections from other irregularities such as galleries and caverns near the tunnel and from partially-closed valves are easily discarded due to their known locations. The air pocket sources have a different pressure feature than a gradual drop of wall stiffness. Finally, leaks have a wave print characterized by steep front reflections that cannot be identified by the FFT approach and thus do not affect the accuracy of the method.

In the ongoing research, the practical application of the proposed method will be tested through a series of in situ measurements conducted on the pressure shaft of a pumped-storage power plant in Switzerland. These measurements will give additional information on the steepness, energy and attenuation of water-hammer waves generated during start-up and shut-down of pumps and turbines.

\section{Acknowledgements}

The study is part of the research project HydroNet for the design, manufacture and operation of pumped storage plants funded by the Swiss Competence Center Energy and Mobility (CCEM$\mathrm{CH}$ ), the Swiss Electrical Research and the Swiss Office for Energy. The authors wish to acknowledge the financial support of the Lombardi Foundation and thank Prof. Didia Covas for her valuable comments during her stay at LCH-EPFL.

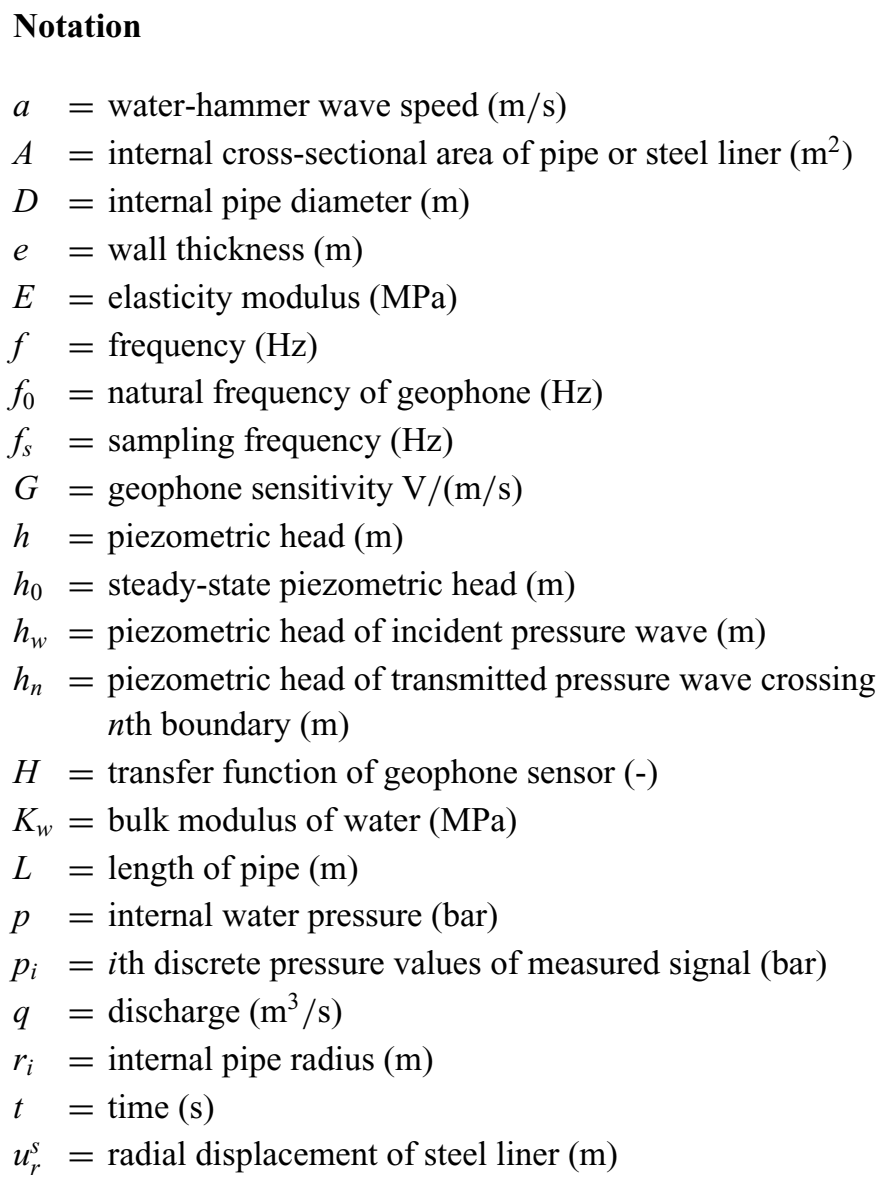

$v=$ geophone output signal $(\mathrm{V})$

$v_{0}=$ steady-state flow velocity $(\mathrm{m} / \mathrm{s})$

$\rho_{w}=$ water density $\left(\mathrm{kg} / \mathrm{m}^{3}\right)$

$\tau_{w}=$ shear stress at water-wall interface $(\mathrm{Pa})$

$\xi=$ damping ratio of geophone (-)

\section{References}

Bergant, A., Simpson, A.R., Vitkovsky, J. (2001). Developments in unsteady pipe low friction modeling. J. Hydraulic Res. 39(3), 249-257.

Bergant, A., Tijsseling, A., Vitkovsky, J., Covas, D., Simpson, A., Lambert, M. (2008a). Parameters affecting water-hammer wave attenuation, shape and timing 1: Mathematical tools. $J$. Hydraulic Res. 46(3), 373-381.

Bergant, A., Tijsseling, A., Vitkovsky, J., Covas, D., Simpson, A., Lambert, M. (2008b). Parameters affecting waterhammer wave attenuation, shape and timing 2: Case studies. J. Hydraulic Res. 46(3), 382-391.

Covas, D., Stoianov, I., Mano, J.F., Ramos, H., Graham, N., Maksimovic, C. (2004). The dynamic effect of pipe-wall viscoelasticity in hydraulic transients 1: Experimental analysis and creep characterization. J. Hydraulic Res. 42(5), 516-530.

Covas, D., Ramos, H., Betâmio de Almeida, A. (2005). Standing wave difference method for leak detection in pipeline systems. J. Hydraulic Eng. 131(12), 1106-1116.

Ferrante, M., Brunone, B. (2003). Pipe system diagnosis and leak detection by unsteady-state tests 2: Wavelet analysis. Advances in Water Res. 26(1), 107-116.

Ghidaoui, M., Zhao, M., Axworthy, D.H., McInnis, D.A. (2005). A review of water hammer theory and practice. Applied Mechanics Reviews 58(1-6), 49-75.

Hachem, F. (2011). Monitoring of steel-lined pressure shafts considering water-hammer wave signals and fluid-structure interaction. PhD thesis No 5171. Ecole Polytechnique Fédérale de Lausanne (EPFL), Switzerland.

Hachem, F., Schleiss, A. (2010). Influence of local stiffness of conduits on water hammer propagation signal. Proc. 1st European IAHR Congress Edinburgh. www.iahr.net.

Hachem, F., Schleiss, A. (2011). A review of wave celerity in frictionless and axisymmetrical steel-lined pressure tunnel. $J$. Fluids and Structures 27(2), 311-328.

Hachem, F., Schleiss, A. (2012). Detection of local wall stiffness drop in steel-lined pressure tunnels and shafts of hydroelectric power plants using steep pressure wave excitation and wavelet decomposition. J. Hydraulic Eng. DOI: 10.1061/(ASCE)HY.1943-7900.0000478.

Hunaidi, O. (2006). A new system for locating leaks in urban water distribution pipes. Management of Env. Quality 17(4), 450-466.

Lang, F.H. (1987). Correlation techniques. Van Nostrand, Princeton, NJ. 
Lavooij, C.S.W., Tijsseling, A.S. (1991). Fluid-structure interaction in liquid-filled piping systems. J. Fluids and Structures 5(5), 573-595.

Mallat, S.G. (1990). A wavelet tour of signal processing. Academic Press, San Diego CA.

Mays, L.W. (2000). Water distribution systems handbook. McGraw-Hill, New York.

Stephens, M.L., Simpson, A.R., Lambert, M.F. (2008). Internal wall condition assessment for water pipelines using inverse transient analysis. Proc. 10th Annual Symp. Water
Distribution Systems Analysis Kruger National Park SA. ASCE, Reston VA (CD-Rom).

Tijsseling, A.S. (2007). Water hammer with fluid-structure interaction in thick-walled pipes. Computer and Structures 85(11-14), 844-851.

Vardy, A.E., Hwang, K.L., Brown, J.M.B. (1993). A weighting model of transient turbulent pipe friction. J. Hydraulic Res. 31(4), 533-548.

Wylie, E.B., Suo, L., Streeter, V.L. (1993). Fluid transients in systems. Prentice Hall, Englewood Cliffs, NJ. 